\title{
Predicting VO2 peak from Six Minute Walk Test in Patients with Pulmonary Hypertension
}

Dario Fuentes ${ }^{1}$, Augusto G. Zapico ${ }^{2}$, Francisco J. Calderon ${ }^{1}$, Erika B. Rosenzweig ${ }^{3}$, Miguel A. Rojo-Tirado ${ }^{1}$, Robert P. Garofano ${ }^{3}{ }^{1}{ }^{\text {Universidad }}$ Politecnica de Madrid, Madrid, Spain. ${ }^{2}$ Universidad Complutense de Madrid, Madrid, Spain. ${ }^{3}$ Columbia University, New York, NY.

(No relationships reported)

Cardiopulmonary Exercise Test (CPET) and Six-minute walk test (6MWT) are important tools in the evaluation and follow up of patients with Idiopathic Pulmonary Arterial Hypertension (IPAH).

PURPOSE: To determine if the simple Six-minute walk test (6MWT) is a valid predictor of VO2 peak in patients with IPAH.

METHODS: 157 patients being treated for IPAH from 2003 to 2013 were included in this study. A 6MWT and a Cardiopulmonary Exercise Test (CPET) were performed as part of

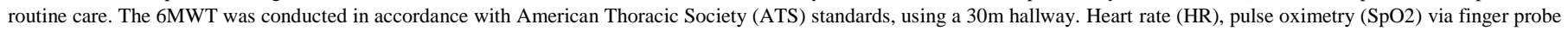
and Borg's rate of perceived exertion (RPE) were measured at baseline and post-test. The CPET was performed on a cycle ergometer using a ramp protocol. Electrocardiogram (EKG), analysis of expired air, SpO2, and blood pressure (BP) were monitored throughout the CPET. Subjects were stratified post-hoc by age $(26$ children=6-12 mean age $9.96 \pm 1.66,73 \%$

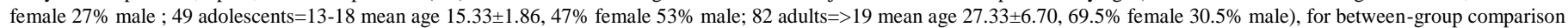
of anthropometric and 6MWT variables. Multiple linear regression analysis was performed to predict VO2 peak for each of the groups from the 6MWT.

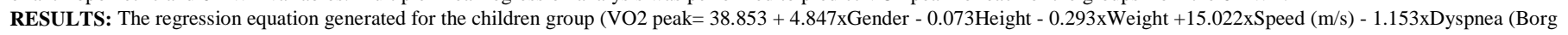
1-10) $-0.175 \times$ Resting HR) was the only model to significantly predict VO2 peak ( $2=0.80, \mathrm{p}=0.804)$. Similar models for the adolescent and adult groups did not achieve statistical significance $(\mathrm{r} 2=0.39, \mathrm{p}=0.142$ and $\mathrm{r} 2=0.38, \mathrm{p}=0.025$, respectively)

CONCLUSION: The measured VO2 peak of the patient population varied greatly by age. A 6MWT was able to accurately predict VO2 peak in children (6-12 years) with IPAH, however the model failed to predict VO2 peak in older populations. These findings suggest that CPET measured VO2 peak is the only reliable method for the evaluation of all patients with IPAH. 